\title{
Acknowledgement to Reviewers of Magnetochemistry in 2018
}

\author{
Magnetochemistry Editorial Office \\ MDPI, St. Alban-Anlage 66, 4052 Basel, Switzerland \\ Published: 24 January 2019
}

\begin{abstract}
Rigorous peer-review is the corner-stone of high-quality academic publishing. The editorial team greatly appreciates the reviewers who contributed their knowledge and expertise to the journal's editorial process over the past 12 months. In 2018, a total of 54 papers were published in the journal, with a median time to first decision of 16 days and a median time to publication of 40.5 days. The editors would like to express their sincere gratitude to the following reviewers for their cooperation and dedication in 2018:
\end{abstract}

\begin{tabular}{ll} 
Afkhami, Shahriar & Feld, Artur \\
Albella, Pablo & Ferrage, Fabien \\
Autschbach, Jochen & Fontanesi, Claudio \\
Baryshnikov, Glib & Fraga-García, Paula \\
Baumgarten, Martin & Friese, Karen \\
Bayraktar, Emin & Frotscher, Martin \\
Bernot, Kevin & Gai, Zheng \\
Bessais, Lotfi & Gaudry, Émilie \\
Bingol, Kerem & Gómez García, Carlos J. \\
Bogani, Lapo & Gooley, Paul \\
Bolvin, Hélène & Goroncy, Alexander \\
Bossmann, Stefan & Graiff, Claudia \\
Bronisz, Robert & Griffiths, Ian \\
Brutscher, Bernhard & Hamlin, Nathaniel \\
Carrella, Luca & Hansen, D. Flemming \\
Carretta, Pietro & Haouas, Mohamed \\
Chi, Celestine N. & Heise, Henrike \\
Chikan, Viktor & Hörner, Gerald \\
Colacio, E. & Huber, David L. \\
Concas, Giorgio & Ishida, Takayuki \\
Constantin, Lucian & Ishikawa, Ryuta \\
De La Venta, Jose & Johansson, Christer \\
Demir, Selvan & Kadela-Tomanek, Monika \\
Di Bella, Santo & Kajiwara, Takashi \\
Drobny, Gary & Kataev, Vladislav \\
Droghetti, Andrea & Kataoka, Yusuke \\
Egodawatte, Shani & Katoh, Keiichi \\
\hline & \\
\hline
\end{tabular}


Kharel, Parashu

Kim, Iltai (Isaac)

Kitazawa, Takafumi

Kitchen, Jonathan

Klug, Candice S.

Kocharian, Armen

Kostakis, George

Koumoulis, Dimitrios

Kumashiro, Kristin

Kuzhir, Pavel

Kuźnik, Nikodem

Kwon, Young-Wan

Kyritsis, Panayotis

Lee, Donghan

Li, Gaojin

Linert, Wolfgang

Liu, Jinming

Long, Gary J.

Mackereth, Cameron

Maesato, Mitsuhiko

Mahata, Manoj Kumar

Marcos, Jorge Sánchez

Martínez-Lillo, José

Massoud, Salah

Matsunaga, Daiki

Mazzei, Pierluigi

Medici, Serenella

Milas, Haralampos N.

Mogi, Iwao

MOLNAR, Gabor

Mota, Antonio J.

Moya, Xavier

Müller, Danny

Murray, Keith

Murray, Keith

Nieto, Pedro

Nishida, Masakazu

Ohara, Keishi

Okubo, Takashi

Pandey, Sudip
Papadimitriou, Vassiliki

Parisi, Filippo

Paul-Boncour, Valérie

Perlepes, Spyros P

Petrakis, Panos. V.

Philipp, Manfred

Phonsri, Wasinee

Pointillart, Fabrice

Power, Philip P.

Rhen, Fernando M. F.

Rigamonti, Luca

Rinehart, Jeffrey

Romero-Muñiz, Carlos

Rossi, Paolo

Sacher, Edward

Saharan, Lalita

Sarioglu, A. Fatih

Sato, Osamu

Scott, Hayley S.

Seidel, Rüdiger W.

Senthil Kumar, Kuppusamy

Slageren, Joris Van

Smith, Graham

Smith, Mark

Stern Taulats, Enric

Tamura, Ryo

Tong, Sheng

Tsai, Hui-Lien

Tsukerblat, Boris

Ukleev, Victor

Vaara, Juha

Van Den Heuvel, Willem

Vargas, Zulema

Vlachos, Nicholas

Walsh, James

Williamson, Mike P

Wolny, Juliusz

Yang, Chen-I

Yoshioka, Naoki

(C) 2019 by the author. Licensee MDPI, Basel, Switzerland. This article is an open access article distributed under the terms and conditions of the Creative Commons Attribution (CC BY) license (http://creativecommons.org/licenses/by/4.0/). 